\title{
A PAGE ON EISENSTEIN SERIES IN RAMANUJAN'S LOST NOTEBOOK
}

\author{
BRUCE C. BERNDT ${ }^{1}$ and AE JA YEE ${ }^{2}$ \\ Department of Mathematics, University of Illinois, 1409 West Green Street, Urbana, IL 61801, USA \\ e-mail:berndt@math.uiuc.edu,yee@math.uiuc.edu
}

(Received 23 October, 2001; accepted 22 February, 2002)

IN MEMORY OF ROBERT A. RANKIN

\begin{abstract}
Page 188 in Ramanujan's lost notebook is devoted to a certain class of infinite series connected with Euler's pentagonal number theorem. These series are represented in terms of Ramanujan's famous Eisenstein series $P, Q$, and $R$. The purpose of this paper is to prove all the formulas on page 188 and to show that one of them leads to an interesting, new recurrence formula for $\sigma(n)$, the sum of the positive divisors of $n$.
\end{abstract}

2000 Mathematics Subject Classification. 11F11.

1. Introduction. On page 188 of his lost notebook, in the pagination of [9], Ramanujan examines the series,

$$
T_{2 k}:=T_{2 k}(q):=1+\sum_{n=1}^{\infty}(-1)^{n}\left\{(6 n-1)^{2 k} q^{n(3 n-1) / 2}+(6 n+1)^{2 k} q^{n(3 n+1) / 2}\right\}, \quad|q|<1 .
$$

Note that the exponents $n(3 n \pm 1) / 2$ are the generalized pentagonal numbers. Ramanujan records formulas for $T_{2 k}, k=1,2, \ldots, 6$, in terms of the Eisenstein series,

$$
\begin{aligned}
P(q) & :=1-24 \sum_{k=1}^{\infty} \frac{k q^{k}}{1-q^{k}}, \\
Q(q) & :=1+240 \sum_{k=1}^{\infty} \frac{k^{3} q^{k}}{1-q^{k}}
\end{aligned}
$$

and

$$
R(q):=1-504 \sum_{k=1}^{\infty} \frac{k^{5} q^{k}}{1-q^{k}}
$$

where $|q|<1$. Ramanujan's formulations of these formulas are cryptic. The first is given

\footnotetext{
${ }^{1}$ Research partially supported by grant MDA904-00-1-0015 from the National Security Agency.

${ }^{2}$ Research partially supported by a grant from the Number Theory Foundation.
} 
by Ramanujan in the form

$$
\frac{1-5^{2} q-7^{2} q^{2}+\cdots}{1-q-q^{2}+\cdots}=P
$$

In succeeding formulas, only the first two terms of the numerator are given, and in two instances the denominator is replaced by a dash - . At the bottom of the page, he gives the first five terms of a general formula for $T_{2 k}$.

The purpose of this paper is to prove these seven formulas and one corollary. Keys to our proofs are the pentagonal number theorem [2, p. 36, Entry 22 (iii)]

$$
(1-q)\left(1-q^{2}\right)\left(1-q^{3}\right) \cdots=:(q ; q)_{\infty}=1+\sum_{n=1}^{\infty}(-1)^{n}\left\{q^{n(3 n-1) / 2}+q^{n(3 n+1) / 2}\right\},
$$

where $|q|<1$, and Ramanujan's famous differential equations [6], [7, p. 142]

$$
q \frac{d P}{d q}=\frac{P^{2}-Q}{12}, \quad q \frac{d Q}{d q}=\frac{P Q-R}{3}, \quad \text { and } \quad q \frac{d R}{d q}=\frac{P R-Q^{2}}{2} .
$$

We now state Ramanujan's six formulas for $T_{2 k}$ followed by a corollary and his general formula. then

THeOrem 1.1. If $T_{2 k}$ is defined by (1.1) and $P, Q$, and $R$ are defined by (1.2)-(1.4),

(i) $\frac{T_{2}(q)}{(q ; q)_{\infty}}=P$,

(ii) $\frac{T_{4}(q)}{(q ; q)_{\infty}}=3 P^{2}-2 Q$,

(iii) $\frac{T_{6}(q)}{(q ; q)_{\infty}}=15 P^{3}-30 P Q+16 R$,

(iv) $\frac{T_{8}(q)}{(q ; q)_{\infty}}=105 P^{4}-420 P^{2} Q+448 P R-132 Q^{2}$,

(v) $\frac{T_{10}(q)}{(q ; q)_{\infty}}=945 P^{5}-6300 P^{3} Q+10080 P^{2} R-5940 P Q^{2}+1216 Q R$,

and

(vi) $\frac{T_{12}(q)}{(q ; q)_{\infty}}=10395 P^{6}-103950 P^{4} Q+221760 P^{3} R-196020 P^{2} Q^{2}$

$$
+80256 P Q R-2712 Q^{3}-9728 R^{2} .
$$

The first formula has an interesting arithmetical interpretation.

COROllary 1.2. For $n \geq 1$, let $\sigma(n)=\sum_{d \mid n} d$, and define $\sigma(0)=-\frac{1}{24}$. Let $n$ denote a non-negative integer. Then

$$
-24 \sum_{\substack{j+k(3 k \pm 1) / 2=n \\ j, k \geq 0}}(-1)^{k} \sigma(j)= \begin{cases}(-1)^{r}(6 r-1)^{2}, & \text { if } n=r(3 r-1) / 2, \\ (-1)^{r}(6 r+1)^{2}, & \text { if } n=r(3 r+1) / 2, \\ 0, & \text { otherwise. }\end{cases}
$$


Since $\sigma(j)$ is multiplicative, we note that $\sigma(j)$ is even except when $j$ is a square or twice a square. Thus, from Corollary 1.2, we see that, unless $n=r(3 r \pm 1) / 2$, the number of representations of $n$ as a sum of a square or twice a square and a generalized pentagonal number $k(3 k \pm 1) / 2$ is even. For example, if $n=20$, then $20=8+12=18+2$.

THEOREM 1.3. Define the polynomials $f_{2 k}(P, Q, R), k \geq 1$, by

$$
f_{2 k}(P, Q, R):=\frac{T_{2 k}(q)}{(q ; q)_{\infty}} .
$$

Then, for $k \geq 1$,

$$
\begin{aligned}
f_{2 k}(P, Q, R)= & 1 \cdot 3 \cdots(2 k-1)\left\{P^{k}-\frac{k(k-1)}{3} P^{k-2} Q+\frac{8 k(k-1)(k-2)}{45} P^{k-3} R\right. \\
& -\frac{11 k(k-1)(k-2)(k-3)}{210} P^{k-4} Q^{2} \\
& \left.+\frac{152 k(k-1)(k-2)(k-3)(k-4)}{14175} P^{k-5} Q R+\cdots\right\} .
\end{aligned}
$$

The statement of Theorem 1.3 is admittedly incomplete. The missing terms represented by $+\cdots$ contain all further products $P^{a} Q^{b} R^{c}$, such that $2 a+4 b+6 c=2 k$. It would be extremely difficult to find a general formula for $f_{2 k}(P, Q, R)$ which would give explicit representations for each coefficient of $P^{2 a} Q^{4 b} R^{6 c}$.

In Section 2 we provide proofs of the two theorems and corollary. In the third section, we offer remarks and related references.

2. Proofs. Important in our proofs are the simple identities

$$
(6 n \pm 1)^{2}=24 \frac{n(3 n \pm 1)}{2}+1
$$

Proof of Theorem 1.1. Observe that

$$
\begin{aligned}
P(q) & =1+24 q \frac{d}{d q} \sum_{n=1}^{\infty} \log \left(1-q^{n}\right) \\
& =1+24 q \frac{d}{d q} \log (q ; q)_{\infty} \\
& =1+24 q \frac{\frac{d}{d q}(q ; q)_{\infty}}{(q ; q)_{\infty}} .
\end{aligned}
$$

Thus, using (1.5) and (2.1), we find that

$$
\begin{aligned}
(q ; q)_{\infty} P(q) & =(q ; q)_{\infty}+24 q \frac{d}{d q}\left(1+\sum_{n=1}^{\infty}(-1)^{n}\left\{q^{n(3 n-1) / 2}+q^{n(3 n+1) / 2}\right\}\right) \\
& =(q ; q)_{\infty}+24 \sum_{n=1}^{\infty}(-1)^{n}\left\{\frac{n(3 n-1)}{2} q^{n(3 n-1) / 2}+\frac{n(3 n+1)}{2} q^{n(3 n+1) / 2}\right\}
\end{aligned}
$$




$$
\begin{aligned}
& =(q ; q)_{\infty}+\sum_{n=1}^{\infty}(-1)^{n}\left\{\left((6 n-1)^{2}-1\right) q^{n(3 n-1) / 2}+\left((6 n+1)^{2}-1\right) q^{n(3 n+1) / 2}\right\} \\
& =(q ; q)_{\infty}+\sum_{n=1}^{\infty}(-1)^{n}\left\{(6 n-1)^{2} q^{n(3 n-1) / 2}+(6 n+1)^{2} q^{n(3 n+1) / 2}\right\}-(q ; q)_{\infty}+1 \\
& =T_{2}(q) .
\end{aligned}
$$

This completes the proof of (i).

In the proofs of the remaining identities of Theorem 1.1, in each case, we apply the operator $24 q \frac{d}{d q}$ to the preceding identity. In each proof we also use the identities

$$
24 q \frac{d}{d q} T_{2 k}(q)=T_{2 k+2}(q)-T_{2 k}(q)
$$

which follows from differentiation and the use of (2.1), and

$$
24 q \frac{d}{d q}(q ; q)_{\infty}=T_{2}(q)-(q ; q)_{\infty}
$$

which arose in the proof of (2.2).

We now prove (ii). Applying the operator $24 q \frac{d}{d q}$ to (2.2) and using (2.3) and (2.4), we deduce that

$$
P(q)\left(T_{2}(q)-(q ; q)_{\infty}\right)+(q ; q)_{\infty} 24 q \frac{d}{d q} P(q)=T_{4}(q)-T_{2}(q) .
$$

Employing (i) to simplify and using the first differential equation in (1.6), we arrive at

$$
P^{2}(q)(q ; q)_{\infty}+2\left(P^{2}(q)-Q(q)\right)(q ; q)_{\infty}=T_{4}(q)
$$

or

$$
T_{4}=\left(3 P^{2}-2 Q\right)(q ; q)_{\infty}
$$

as desired.

To prove (iii), we apply the operator $24 q \frac{d}{d q}$ to (2.5) and use (2.3) and (2.4) to deduce that

$$
\begin{aligned}
T_{6}-T_{4} & =24\left(6 P q \frac{d P}{d q}-2 q \frac{d Q}{d q}\right)(q ; q)_{\infty}+\left(3 P^{2}-2 Q\right)\left(T_{2}-(q ; q)_{\infty}\right) \\
& =\left(12 P\left(P^{2}-Q\right)-16(P Q-R)\right)(q ; q)_{\infty}+\left(3 P^{2}-2 Q\right)(P-1)(q ; q)_{\infty},
\end{aligned}
$$

where we used (1.6) and (i). If we now employ (2.5) and simplify, we conclude that

$$
T_{6}=\left(15 P^{3}-30 P Q+16 R\right)(q ; q)_{\infty} .
$$

In general, by applying the operator $24 q \frac{d}{d q}$ to $T_{2 k}$ and using (2.3) and (2.4), we find that

$$
T_{2 k+2}-T_{2 k}=24 q \frac{d}{d q} f_{2 k}(P, Q, R)(q ; q)_{\infty}+f_{2 k}(P, Q, R)(P-1)(q ; q)_{\infty},
$$


where we have used the notation (1.8). Then proceeding by induction while using the formula (1.8) for $T_{2 k}$, we find that

$$
\frac{T_{2 k+2}}{(q ; q)_{\infty}}=24 q \frac{d}{d q} f_{2 k}(P, Q, R)+P f_{2 k}(P, Q, R) .
$$

Thus, in the notation (1.8),

$$
f_{2 k+2}(P, Q, R)=24 q \frac{d}{d q} f_{2 k}(P, Q, R)+P f_{2 k}(P, Q, R) .
$$

With the use of (2.6) and the differential equations (1.6), it should now be clear how to prove the remaining identities, (iv)-(vi), and so we omit further details.

Proof of Corollary 1.2. By expanding the summands of $P(q)$ in (1.2) in geometric series and collecting the coefficients of $q^{n}$ for each positive integer $n$, we find that

$$
P(q)=1-24 \sum_{n=1}^{\infty} \sigma(n) q^{n}=-24 \sum_{n=0}^{\infty} \sigma(n) q^{n}
$$

upon using the definition $\sigma(0)=-\frac{1}{24}$. Thus, by (1.5), Theorem 1.1 (i) can be written in the form

$$
\begin{aligned}
& -24 \sum_{j=0}^{\infty} \sigma(j) q^{j} \cdot\left(1+\sum_{k=1}^{\infty}(-1)^{k}\left\{q^{k(3 k-1) / 2}+q^{k(3 k+1) / 2}\right\}\right) \\
& =1+\sum_{n=1}^{\infty}(-1)^{n}\left\{(6 n-1)^{2} q^{n(3 n-1) / 2}+(6 n+1)^{2} q^{n(3 n+1) / 2}\right\} .
\end{aligned}
$$

Equating coefficients of $q^{n}, n \geq 1$, on both sides of (2.7), we complete the proof.

Proof of Theorem 1.3. We apply induction on $k$. For $k=1,2$, the assertion (1.9) is true by Theorem 1.1 (i), (ii). Assume therefore that (1.9) is valid; we shall prove (1.9) for $k$ replaced by $k+1$. Our proof employs (2.6).

The terms involving $P^{k-6}$, which are not displayed on the right side of (1.9), are of the forms $c_{1} P^{k-6} R^{2}, c_{2} P^{k-6} Q^{3}$, and $c_{3} P^{k-6} R Q^{2}$, for certain constants $c_{1}, c_{2}$, and $c_{3}$. If we differentiate each of these expressions and use the differential equations (1.6), we can easily check that no terms like the five displayed forms in (1.9) arise. Thus, when applying (2.6) along with induction on $k$, we need only concern ourselves with the derivatives of the five displayed terms in (1.9); no further contributions are made by the derivatives of undisplayed terms to the five coefficients with $k$ replaced by $k+1$. 
By (2.6), (1.6), and induction, we find that

$$
\begin{aligned}
& f_{2 k+2}(P, Q, R)=1 \cdot 3 \cdots(2 k-1)\left\{k P^{k-1} \cdot 2\left(P^{2}-Q\right)\right. \\
& -\frac{k(k-1)(k-2)}{3} P^{k-3} Q \cdot 2\left(P^{2}-Q\right)-\frac{k(k-1)}{3} P^{k-2} \cdot 8(P Q-R) \\
& +\frac{8 k(k-1)(k-2)(k-3)}{45} P^{k-4} R \cdot 2\left(P^{2}-Q\right) \\
& +\frac{8 k(k-1)(k-2)}{45} P^{k-3} \cdot 12\left(P R-Q^{2}\right) \\
& -\frac{11 k(k-1)(k-2)(k-3)(k-4)}{210} P^{k-5} Q^{2} \cdot 2\left(P^{2}-Q\right) \\
& -\frac{11 k(k-1)(k-2)(k-3)}{210} P^{k-4} \cdot 2 Q \cdot 8(P Q-R) \\
& +\frac{152 k(k-1)(k-2)(k-3)(k-4)(k-5)}{14175} P^{k-6} Q R \cdot 2\left(P^{2}-Q\right) \\
& +\frac{152 k(k-1)(k-2)(k-3)(k-4)}{14175} P^{k-5} R \cdot 8(P Q-R) \\
& \left.+\frac{152 k(k-1)(k-2)(k-3)(k-4)}{14175} P^{k-5} Q \cdot 12\left(P R-Q^{2}\right)+\cdots\right\} \\
& +1 \cdot 3 \cdots(2 k-1)\left\{P^{k+1}-\frac{k(k-1)}{3} P^{k-1} Q+\frac{8 k(k-1)(k-2)}{45} P^{k-2} R\right. \\
& -\frac{11 k(k-1)(k-2)(k-3)}{210} P^{k-3} Q^{2} \\
& \left.+\frac{152 k(k-1)(k-2)(k-3)(k-4)}{14175} P^{k-4} Q R+\cdots\right\} \text {. }
\end{aligned}
$$

The remaining task is to collect coefficients of the five terms, $P^{k+1}, P^{k-1} Q$, $P^{k-2} R, P^{k-3} Q^{2}$, and $P^{k-4} Q R$. Upon completing this routine, but admittedly tedious, task, we complete the proof of the theorem as stated by Ramanujan in [9].

3. Further remarks. Beginning with his paper [6] and notebooks [8], Ramanujan devoted considerable attention to Eisenstein series, most notably to $P, Q$, and $R$, defined by (1.2)-(1.4). In particular, see [1, pp. 318-333], [2, Chapters 16, 17], and [3, Chapter $33]$. The identities in $[2$, pp. 59, 61-65] are particularly related to the ones proved here. His lost notebook [9] contains several new results on $P, Q$, and $R$, including those proved in this paper. A survey of Ramanujan's work on Eisenstein series, especially the claims in his lost notebook, has been written by the authors [4].

The functions $Q$ and $R$ can be represented or evaluated in terms of parameters prominent in the the theory of elliptic functions [2, pp. 126-127]. The function $P$ does have one representation in terms of elliptic function parameters $[2$, p. 120, Entry 9 (iv)], but it is in terms of $d z / d x$, where $z:=z(x):={ }_{2} F_{1}\left(\frac{1}{2}, \frac{1}{2} ; 1 ; x\right)$, and where $q:=\exp (-\pi z(1-x) / z(x))$. The appearance of $d z / d x$ greatly decreases the formula's usefulness. Evaluations of $Q$ and $R$ can be given in terms of $z ; d z / d x$ does not appear. Perhaps the representation of $P$ given in Theorem 1.1 (i) will prove to be more useful than the aforementioned representation for $P$. While $Q$ and $R$ are modular forms, $P$ is 
not, and for this reason it does not share many properties and representations that $Q$ and $R$ possess.

Besides Corollary 1.2, other identities of Ramanujan can be reformulated in terms of divisor sums $\sigma_{k}(n):=\sum_{d \mid n} d^{k}$. In particular, see [1, pp. 326-329] and the references cited there. However, by far, the most comprehensive study of identities of this sort has been undertaken by J. G. Huard, Z. M. Ou, B. K. Spearman, and K. S. Williams [5], where many references to the literature can also be found. On the other hand, R. A. Rankin [10] used elementary identities for divisor sums to establish relations between Eisenstein series. In particular, he proved Ramanujan's differential equations (1.6) along these lines.

Acknowledgement. The authors thank Heng Huat Chan for helpful remarks and Alexandru Zaharescu for the observation after Corollary 1.2.

\section{REFERENCES}

1. B. C. Berndt, Ramanujan's Notebooks, Part II (Springer-Verlag, 1989).

2. B. C. Berndt, Ramanujan's Notebooks, Part III (Springer-Verlag, 1991).

3. B. C. Berndt, Ramanujan's Notebooks, Part V (Springer-Verlag, 1998).

4. B. C. Berndt and A. J. Yee, Ramanujan's contributions to Eisenstein series, especially in his lost notebook, in Number theoretic methods - future trends (eds. C. Jia and S. Kanemitsu), (Kluwer, Dordrecht, 2002), 31-53.

5. J. G. Huard, Z. M. Ou, B. K. Spearman, and K. S. Williams, Elementary evaluation of certain convolution sums involving divisor functions, in Number Theory for the Millennium, Vol. 2 (eds. M. A. Bennett, B. C. Berndt, N. Boston, H. G. Diamond, A. J. Hildebrand, and W. Philipp), (AK Peters, 2002), 229-274.

6. S. Ramanujan, On certain arithmetical functions, Trans. Cambridge Philos. Soc. 22 (1916), 159-184.

7. S. Ramanujan, Collected papers, Cambridge University Press, Cambridge, 1927; reprinted by Chelsea, New York, 1962; reprinted by the American Math. Soc., Providence, RI, 2000.

8. S. Ramanujan, Notebooks (2 volumes) (Tata Institute of Fundamental Research, Bombay, 1957). 1988).

9. S. Ramanujan, The lost notebook and other unpublished papers (Narosa, New Delhi,

10. R. A. Rankin, Elementary proofs of relations between Eisenstein series, Proc. Roy. Soc. Edinburgh Sect. A 76 (1976), 107-117. 